\title{
Impactos de variáveis climáticas na agilidade de bezerros Nelore neonatos
}

\author{
Impacts of climatic variable in the agility of neonate Nelore \\ Luciandra Macedo ToledoI Mateus José Rodrigues Paranhos da CostaII \\ Evaldo Antonio Lencioni Titto ${ }^{\mathrm{III}}$ Leopoldo de Andrade Figueiredo ${ }^{\mathrm{IV}}$ \\ Denise de Souza Ablas ${ }^{\text {III }}$
}

\section{RESUMO}

Com os objetivos de identificar os padrões comportamentais nas relações materno-filiais de bovinos da raça Nelore, de entender as interferências ambientais nesta relação e de propor soluções práticas de manejo, foram realizadas medidas de comportamento das atividades de 112 vacas e seus bezerros nas primeiras horas após o parto, em duas propriedades situadas em Sertãozinho e Brotas, no Estado de São Paulo. Foram analisadas as variáveis: tempo para ficar em pé (LP) e tempo para mamar (LM), em minutos, nos bezerros e tempo de contato com a cria (TCC) e tempo deitada (TD), em porcentagem, nas vacas. Foram registradas a temperatura e a umidade relativa do ar para o cálculo do índice de temperatura e umidade do ar (ITU), correlacionandose esse índice com as variáveis comportamentais. Os resultados mostraram efeitos significativos $(P<0,05)$ de propriedade e de local do parto em relação ao rebanho e ao ITU. A LP pode ser uma medida de vigor em bezerros e mostrou-se influenciada por diversos fatores ambientais. O TCC teve efeito significativo $(P<0,05)$ na LP. A seleção de bovinos de corte deve incluir as características de agilidade dos bezerros recém-nascidos e do cuidado maternal das vacas como ferramentas para otimização e viabilidade econômica dessa exploração.

Palavras-chave: ambiente de parto, comportamento materno, agilidade em bezerros recém-nascidos.

\section{ABSTRACT}

Aiming at identifing the behavioral patterns in the cow-calf relationship of the Nelore beef breed and how the environment can interfere in this relationship, and proposing practical solutions of manage, a study measuring behavior, by means of direct observations of the activities of 112 cows and their calves in the first hours after calving was conducted in two farms located in Sertãozinho and Brotas, State of São Paulo. The variables latency time to stand up (LP) and latency time to suck (LM) in minutes, in the calves, and time to contact the calves (TCC) and lying time (TD), in percentage, in the cows were measured and analyzed. Temperature and the relative humidity of the air were registered to generate the temperature and humidity index (ITU), which was correlated to the cowcalf behavior variables. The results showed significative effects $(P<0.05)$ of farm, location of calving in relation to the herd and ITU. The LP can be a measure of calf vigor, and it was influenced by several environmental factors. The TCC had significant effect $(P<0.05)$ on the LP. Beef cattle selection must include the characteristics of neonate calf agility and maternal care of the cows, as tools to optimization and economical viability of this exploration.

Key words: calving environment, maternal behavior, neonate calf agility.

\section{INTRODUÇÃO}

A ingestão do colostro é importante para a obtenção de carboidratos, lipídeos, proteínas, minerais, vitaminas, hormônios e fatores de crescimento que auxiliarão no desenvolvimento do trato gastrointestinal dos neonatos (BLUM \& HAMMON, 2000) e da imunidade passiva, qu devido ao tipo de formação placentária, não são passados de mãe para filho durante a gestação, devendo ser adquiridos pela ingestão do colostro, através das imunoglobulinas (PIRES et al., 1993; PARANHOS DA COSTA \&

\footnotetext{
IAgência Paulista de Tecnologia Agropecuária, (APTA), Registro, SP, Brasil. E-mail: lmtoledo@aptaregional.sp.gov.br.

IFaculdade de Ciências Agrárias e Veterinárias, Universidade de São Paulo (FCAV/UNESP), Jaboticabal, SP, Brasil.

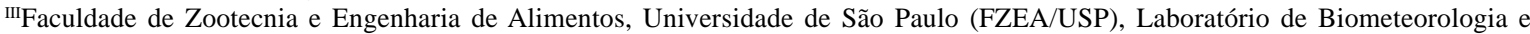
Etologia, CP 23, 13630-000, Pirassununga, SP, Brasil. E-mail: titto@usp.br. Autor para correspondência.

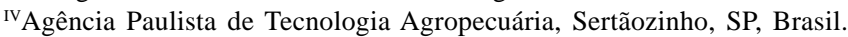


CROMBERG, 1998). Adicionalmente, bezerros bovinos não apresentam, ao nascer, reservas energéticas suficientes para assegurar eficiência no processo de termorregulação (LUCCI, 1989), necessitando ingerir rapidamente o colostro para assegurar sua sobrevivência no ambiente. Com isso, na bovinocultura de corte, com os bezerros criados a pasto ao pé das vacas, a importância do recém-nascido em levantar-se rapidamente e ingerir seu alimento tem levado a estudos de características para seleção de animais mais ágeis (SCHMIDEK, 2003; RILEY et al., 2004). Verifica-se, nos diferentes sistemas de exploração de bovinocultura, a existência de um padrão comportamental nas relações materno-filiais, talvez decorrentes do ambiente predominante durante sua evolução, cabendo aos técnicos e produtores entenderem a importância destes comportamentos e obterem sucesso na exploração de bovinos de corte. Desta forma, os objetivos deste trabalho foram identificar os padrões comportamentais nas relações mães-filhotes de bovinos da raça Nelore, como o ambiente pode interferir e, assim, propor soluções práticas de manejo para os problemas enfrentados.

\section{MATERIAL E MÉTODOS}

Os dados foram colhidos em duas fazendas: Estação Experimental de Zootecnia de Sertãozinho SP (Instituto de Zootecnia, Secretaria de Agricultura e Abastecimento do Estado de São Paulo) e na Fazenda Mundo Novo, situada em Brotas - SP, denominadas propriedades I e II, respectivamente. As duas propriedades adotaram estação de monta, que resulta na concentração de partos entre os meses de agosto a novembro de cada ano na propriedade I e de novembro a janeiro de cada ano na propriedade II. Ambas utilizaram áreas destinadas à maternidade, sendo que, na propriedade I, o piquete maternidade era pequeno ( 0,8 hectares) e a densidade animal era alta, variando no decorrer da estação de nascimento, mas, na época de pico, concentrou cerca de 100 vacas. Nesta mesma propriedade, as vacas recebiam alimento exclusivamente em comedouros, duas vezes ao dia, composto de silagem de milho ou sorgo e, eventualmente, de caroço de algodão, enquanto estavam na maternidade. No restante do ano, a alimentação era somente obtida do pastejo com suplementação mineral. Na propriedade II, a alimentação era obtida exclusivamente das pastagens, em área de 10 hectares, com 50 vacas na época de maior densidade animal. A colheita de dados ocorreu nos anos de 1997 e 1998 na propriedade I e de 1999 na propriedade II, resultando no registro de comportamento de 77 e 35 vacas e bezerros nas propriedades I e II, respectivamente.
Medidas de comportamento

As medidas de comportamento foram realizadas no período diurno, por meio de observações diretas, com colheita instantânea e amostragem focal (MARTIN \& BATESON, 1986), com intervalo amostral de 15 minutos, das atividades de vacas e bezerros nas primeiras cinco horas após o parto. Antes de cada período de observação, era avaliada a confiabilidade inter e intra-observadores para os registros de comportamento. A confiabilidade foi aferida com o uso de vídeos e observações diretas no campo do comportamento materno-filial, em que cada observador registrava os comportamentos de interesse e depois eram comparados os registros, repetindo-se este procedimento até que houvesse unanimidade entre os observadores, sem nenhuma diferença (relativas aos movimentos de vacas e bezerros, como tentar levantar e efetivamente ficar em pé, ou lamber e empurrar a cria) quanto ao registro de um mesmo comportamento. $\mathrm{O}$ acompanhamento de cada vaca iniciava-se quando esta apresentava características que indicavam a iminência do parto, geralmente apresentando secreção intensa ou exposição dos envoltórios fetais. O registro dos dados iniciava-se após a expulsão completa do bezerro, anotando-se a postura corporal e as atividades desenvolvidas pela vaca e por ele. Foram definidas as seguintes categorias comportamentais para vacas e bezerros em relação à postura corporal: deitado (o animal não estava apoiado em nenhum dos membros, podendo ser em decúbito lateral ou ventral); em pé (o animal encontrava-se apoiado sobre os quatro membros); em movimento circular (fazendo movimentos ao redor do eixo do próprio corpo, permanecendo no mesmo local físico); deslocamento (quando o animal mudava os membros de lugar e saia do local que estava antes). Especificamente para as vacas, foram definidas as seguintes categorias relacionadas às atividades pósparto: contato com a cria (atividades que consistiam em cheirar, lamber e empurrar a cria, estando em contato direto); outras atividades (qualquer atividade desenvolvida que não consistia em contato íntimo com o bezerro). Com relação às atividades dos bezerros, foram consideradas também as seguintes categorias: mamando (quando o bezerro estava com o teto na boca, fazendo movimento de sucção); outras atividades (qualquer atividade desenvolvida, exceto a apreensão dos tetos). Os registros terminavam logo após o bezerro conseguir sucesso na primeira mamada ou, quando esta não acontecia, após cinco horas de observação. Todos os partos analisados neste estudo ocorreram naturalmente, sem nenhuma assistência; os casos que necessitaram de ajuda não fizeram parte do banco de dados.

Ciência Rural, v.37, n.5, set-out, 2007. 
As propriedades I e II apresentam clima tropical, caracterizado por inverno seco e verão quente e chuvoso. Os dados meteorológicos de interesse, temperatura e umidade do ar, foram obtidos através de uma estação meteorológica eletrônica na propriedade I e de termohigrógrafo na propriedade II. Estes dois elementos meteorológicos foram utilizados para o cálculo do índice de temperatura e umidade (ITU), criado por THOM (1959) e relacionado ao conforto para animais por KELLY \& BOND (1971), de acordo com a fórmula: $\mathrm{ITU}=\mathrm{TBS}+0,36 \mathrm{To}+41,5$; em que TBS= temperatura de bulbo seco e To= temperatura do ponto de orvalho. O ITU foi utilizado visando a identificar as possíveis influências climáticas sobre o comportamento das vacas e de seus bezerros no momento do parto.

Para algumas análises específicas, os índices obtidos foram agrupados em cinco classes (CITU) de acordo com a amplitude dos dados definida da seguinte forma: 84,68-62,21 $\div 5=4,49$, onde 84,68= ITU máximo, 62,21= ITU mínimo, 5= número de classes arbitrado e $4,49=0$ intervalo de cada classe, resultando nas seguintes classes: $1=<66,70 ; 2=\geq 66,70<71,19 ; 3=\geq$ $71,19<75,69 ; 4=\geq 75,69<80,18$ e $5=\geq 80,18$. Essas faixas de ITU não têm relação com o conforto ou a tolerância térmica que ainda é alvo de volumosa polêmica na literatura.

Foram definidos dois conjuntos de variáveis dependentes, um para o comportamento dos bezerros e outro para o comportamento das vacas. Para os bezerros, foram consideradas duas variáveis: latência para ficar em pé (LP) (tempo, em minutos, que o bezerro leva desde o nascimento até permanecer apoiado nos quatro membros, estando ou não equilibrado); latência para mamar (LM) (definido como o tempo, em minutos, que o bezerro levou para iniciar a apreensão do teto após o nascimento). Para as vacas, as variáveis foram: tempo em contato com a cria (TCC) (porcentagem do tempo total de observação, em minutos, de todas as atividades de cuidado que a vaca realiza estando em íntimo contato com o bezerro, ou seja, cheirando, lambendo ou tocando); tempo deitada (TD) (definido como porcentagem do tempo total de observação, em minutos, das atividades que a vaca estava deitada, executando qualquer atividade). Após o período de observações e registros no campo, os dados foram digitados e as variáveis de interesse calculadas. Quando o bezerro não conseguiu mamar, ou mesmo não conseguiu levantar, no prazo de cinco horas, assumiu-se o valor de 315 minutos para as latências (para ficar em pé e mamar), que foi definido pelo tempo total de observação mais quinze minutos. Isto foi realizado para que fosse possível a utilização de um maior número de informações na análise dos dados, bem como para assegurar que as médias não fossem subestimadas. Assim, foi assumido o valor máximo do tempo de observação como representativo da latência para ficar em pé (LP) e da latência para mamar (LM), quando essas categorias comportamentais levaram mais tempo para ocorrer do que os 300 minutos de observação.

Foram realizadas análises exploratórias com o propósito de caracterizar a forma de distribuição dos dados e as fontes de variação mais relevantes, sendo que, a partir destes resultados, foram definidos os modelos matemáticos para utilização nas análises confirmatórias. As análises estatísticas para as variáveis LP e TCC foram conduzidas pelo procedimento GLM, contido no pacote estatístico SPSS (SPSS, 2004). Na variável LP, os efeitos constantes no modelo foram: propriedade (I e II), local do parto em relação ao rebanho (LPR), sendo essa medida aproximada determinada pela distância conhecida entre os mourões (1= junto: vaca encostada na outra vaca até a distância de 10 metros, 2= próxima: entre 10 e 30 metros e 3= afastada: mais de 30 metros), pela interação propriedade e local do parto em relação ao rebanho (PROP x LPR), pelo sexo do bezerro ( 1 = macho e 2 = fêmea), pelas classes de índice de temperatura e umidade (CITU), pela interação CITU e PROP, pela interação PROP e ano e pela covariável TCC. Para a variável tempo em contato com a cria (TCC), as variáveis dependentes foram propriedade (I e II), sexo do bezerro ( $1=$ macho e $2=$ fêmea), as classes de número de parto (1= primíparas e $2=$ pluríparas), classes de $L P(C L P=1=$ até 30 minutos, $2=$ de 30 até 60 e $3=$ acima de 60 minutos), CITU, a interação CITU e PROP e a interação CITU e CLP. As variáveis LM e TD foram correlacionadas com as demais variáveis comportamentais e meteorológicas. Foram considerados como efeitos significativos $\mathrm{P}<0,05$.

\section{RESULTADOS E DISCUSSÃO}

O resumo do quadro de análise de variância para LP é apresentado na tabela 1. Os resultados mostraram efeitos significativos para PROP, local do parto em relação ao rebanho (LPR), para a interação destas duas (PROP x LPR), para classes do índice de temperatura e umidade (CITU) e para a covariável TCC, desassociada do sexo do bezerro e da interação CITU e PROP, que não foram fontes de variação para LP.

As estimativas das médias para LP e os respectivos erros-padrão foram de 62,15 \pm 7,42 e 97,15 $\pm 17,71$ para as PROP I e II, respectivamente. A LPR mostrou efeito significativo na LP, bem como da interação entre PROP x LPR. As estimativas das médias 
Tabela 1 - Resumo da análise de variância para tempo de latência para ficar em pé (LP), sendo PROP (propriedade), LPR (local do parto em relação ao rebanho), CITU (classe de índice de temperatura e umidade do ar), TCC (tempo em contato com a cria, covariável).

\begin{tabular}{lcl}
\hline Fontes de variação & $\begin{array}{c}\text { Graus de } \\
\text { liberdade }\end{array}$ & $\begin{array}{c}\text { Quadrados } \\
\text { médios }\end{array}$ \\
\hline PROP & 1 & $12371,36^{* *}$ \\
LPR & 2 & $5939,25^{* *}$ \\
PROP * LPR & 2 & $7489,38^{* *}$ \\
Sexo & 1 & $1921,38^{\text {NS }}$ \\
CITU & 4 & $2720,93^{*}$ \\
CITU* PROP & 4 & $880,69^{\text {NS }}$ \\
PROP*ANO & 1 & $63,93^{\text {NS }}$ \\
TCC & 1 & $10772,31^{* *}$ \\
Resíduo & 74 & 1131,03 \\
& \multicolumn{2}{c}{0,458} \\
r $^{2}$ (coeficiente de determinação) & \multicolumn{2}{c}{$45,56 \%$} \\
CV (coeficiente de variação) & \multicolumn{2}{c}{} \\
\hline
\end{tabular}

${ }^{* *} \mathrm{P}<0,01 ;{ }^{*} \mathrm{P}<0,05$ e NS - não-significativo.

para LP, de acordo com LPR, podem ser visualizadas na tabela 2. Na tabela 3 é apresentado o quadro de análise de variância para TCC. Dos efeitos utilizados para a análise da variável TCC, apresentaram significância a CLP e a interação CITU e CLP. Não houve efeito de propriedade, sexo do bezerro, CNP, CITU e das interações PROP * CITU e CITU * CLP. As variáveis LP e LM mostraram correlação significativa e positiva ( $\mathrm{r}=0,756 ; \mathrm{P}<0,01)$, indicando uma forte associação: os animais que levantaram mais rapidamente mamaram também mais rápido e obtiveram menor tempo de latência entre ficar de pé e ingerir o colostro. Essas mesmas variáveis mostraram uma correlação negativa com ITU ( $r=-0,294$ e r= -0,239 para LP e LM; P<0,05), indicando que existe uma associação com menores valores de ITU e maiores LP e LM. Não houve correlação entre as variáveis estudadas para as vacas (TD e TCC) e a variável climática ITU (P>0,05).

Tabela 2 - Estimativa das médias para tempo de latência para ficar em pé (LP), em minutos, e respectivos erros-padrão, de acordo com o local do parto (propriedades I e II) e em relação ao rebanho (LPR: 1-junto, 2-próximo e 3afastado).

\begin{tabular}{|c|c|c|}
\hline \multirow[b]{2}{*}{ LPR } & \multicolumn{2}{|c|}{ LP/PROP } \\
\hline & I & II \\
\hline 1 & $60,61 \pm 7,90$ & $141,24 \pm 15,55$ \\
\hline 2 & $65,99 \pm 10,01$ & $76,84 \pm 10,27$ \\
\hline 3 & $59,83 \pm 13,78$ & $73,37 \pm 17,08$ \\
\hline Médias gerais & $62,15 \pm 7,42$ & $97,15 \pm 17,71$ \\
\hline
\end{tabular}

Do total de bezerros observados (112), apenas três bezerros não mamaram no tempo de registro do comportamento (300 minutos), dois nem chegaram a levantar neste tempo de observação e um levantou em 75 minutos e, aos 105 minutos, procurou os tetos no corpo da mãe.

O índice de temperatura e umidade do ar não mostrou efeito no TCC e apenas a interação entre CITU e LP mostrou afetar o tempo que a vaca passa em contato com o bezerro.

A postura do bezerro pode ser um importante fator no imprinting entre mãe e filho se ocorrer alguma interferência do ambiente. No presente estudo, por exemplo, foram observados dois casos distintos: um, quando começava a chover, todo o rebanho se deslocava e assim a vaca parida queria acompanhar o rebanho, porém o bezerro ainda não havia conseguido levantar-se; outro caso, na ocorrência de qualquer tipo de movimentação anormal no local do parto, como presença de pessoas, veículos e deslocamento de outros animais, a vaca iniciava o deslocamento deste local e mugia para o bezerro que, ainda deitado, não a acompanhava. Nesses casos, os bezerros podem ser abandonados (PARANHOS DA COSTA \& CROMBERG, 1998). Outros casos mostrando a importância do bezerro ficar em pé foram observados, no presente estudo, em nascimento de gêmeos (que não entraram no banco de dados devido ao pequeno número de registros), pois quando um bezerro conseguia levantar e começava o comportamento de procurar tetos no corpo da mãe, o outro quase sempre parava de receber atenção materna. Também se um

Tabela 3 - Resumo da análise de variância para tempo em contato com a cria (TCC), sendo: PROP (propriedade), CNP (classe de número de partos), CLP (classe de latência para ficar em pé), CITU (classe de índice de temperatura e umidade do ar).

\begin{tabular}{lcl}
\hline Fontes de variação & $\begin{array}{c}\text { Graus de } \\
\text { liberdade }\end{array}$ & $\begin{array}{c}\text { Quadrado } \\
\text { médio }\end{array}$ \\
\hline PROP & 1 & $71,07^{\mathrm{NS}}$ \\
SEXO & 1 & $204,99^{\mathrm{NS}}$ \\
CNP & 1 & $138,36^{\mathrm{NS}}$ \\
CLP & 2 & $3408,86^{* *}$ \\
CITU & 4 & $666,83^{\mathrm{NS}}$ \\
CITU * PROP & 4 & $688,86^{\mathrm{NS}}$ \\
CITU * CLP & 8 & $1090,65^{*}$ \\
Resíduo & 72 & 467,46 \\
& & \\
r $^{2}$ (coeficiente de determinação) & 0,45 & \\
CV (coeficiente de variação) & $33,06 \%$ & \\
\hline
\end{tabular}

${ }^{* *} \mathrm{P}<0,01,{ }^{*} \mathrm{P}<0,05$.

Ciência Rural, v.37, n.5, set-out, 2007. 
bezerro era capaz de seguir a vaca, ela não mugia para que o outro a acompanhasse, sendo assim abandonado.

O tempo de latência para ficar em pé pode ser utilizado como medida de vigor ou agilidade, em bezerros, porém mostrou-se influenciado por diversos fatores ambientais, que devem ser levados em consideração quando se pretende comparar desempenhos entre animais.

As diferenças encontradas nas propriedades podem indicar a variação devido ao ambiente que, apesar de consideradas iguais quanto ao sistema de produção, extensivo e alimentação a pasto ou em comedouros, possuem diferentes estratégias de manejo e propósitos específicos de seleção. Assim, as diferenças encontradas entre as propriedades no presente estudo serviram apenas para ilustrar as diferenças nos diversos sistemas de produção.

O efeito significativo do local do parto em relação ao rebanho e da interação com a propriedade sugere as diferenças já descritas acima. Vale ressaltar que o momento logo após o parto é crucial para a formação dos laços entre mãe e filho e esse afastamento do rebanho possibilita a formação dos laços maternofiliais mais sólidos, sem a interferência de outros membros do rebanho (LIDFORS, 1994). Com o uso de estação de monta/parição, a concentração de fêmeas em iminência do parto aumenta e, nesta fase, há um maior interesse e atração por neonatos, devido à ação hormonal responsável por essa motivação, e, assim, não é incomum haver aproximação de outras vacas interessadas em tomar o bezerro recém-nascido de sua mãe e, conseguindo êxito, muitas vezes acabam ocasionando transtornos nos Registros Zootécnicos. Além deste aspecto “de roubo de bezerro", também existe a possibilidade de haver pisoteio do recémnascido devido à ocorrência de partos em áreas de maior movimentação ou densidade do rebanho. Como técnica de manejo, áreas destinadas à maternidade em bovinos de corte muitas vezes localizam-se próximas aos currais e locais movimentados para uma melhor vistoria e, com isso, a possibilidade para o afastamento da fêmea do restante do grupo pode ser menos vantajosa do que sua permanência próxima aos humanos.

No aspecto de ambiente físico, o bezerro sofre com a brusca mudança de temperatura do útero de sua mãe, próxima a $39^{\circ} \mathrm{C}$, para um ambiente muitas vezes 20 a $25^{\circ} \mathrm{C}$ mais baixo (LE DIVIDICH et al., 1992)e, mesmo no Brasil, um país tropical, o bezerro pode sofrer estresse pelo frio. Na literatura, referências encontradas em criações de vacas leiteiras mostraram que valores de índice (ITU) superiores a 70 merecem atenção quanto ao auxílio para dissipação de calor dos animais (TITTO, 1998; BACCARI, 2001); no entanto, os resultados do presente estudo mostraram que bezerros de corte, na fase neonatal, necessitam de valores superiores para o índice de temperatura e umidade. Ao analisar-se os resultados da figura 1, constatar-se que bezerros nascidos quando o ITU foi menor que 66,70 apresentaram tempo de latência para ficar em pé (LP) superior a 100 minutos e significativamente maior que nas outras quatro classes de ITU $(\mathrm{P}<0,05)$. Outros estudos também sugerem que a zona de conforto térmico dos bezerros recém-nascidos situa-se em temperaturas mais altas. PIRES et al. (1993) descreveram o registro de vários autores de que os bezerros são mais letárgicos no inverno, demorando mais tempo para ficarem em pé e, consequentemente, para mamarem.

O tempo que a vaca passa em íntimo contato com o bezerro demonstrou efeito significativo na latência para ficar em pé $(\mathrm{P}<0,01)$, conforme a tabela 3 . Quando analisado como covariável na latência para ficar em pé, este efeito e a correlação entre essas duas variáveis evidenciaram que menores valores de LP, vistos em bezerros considerados mais ágeis e que se movimentaram mais, aumentaram as respostas de

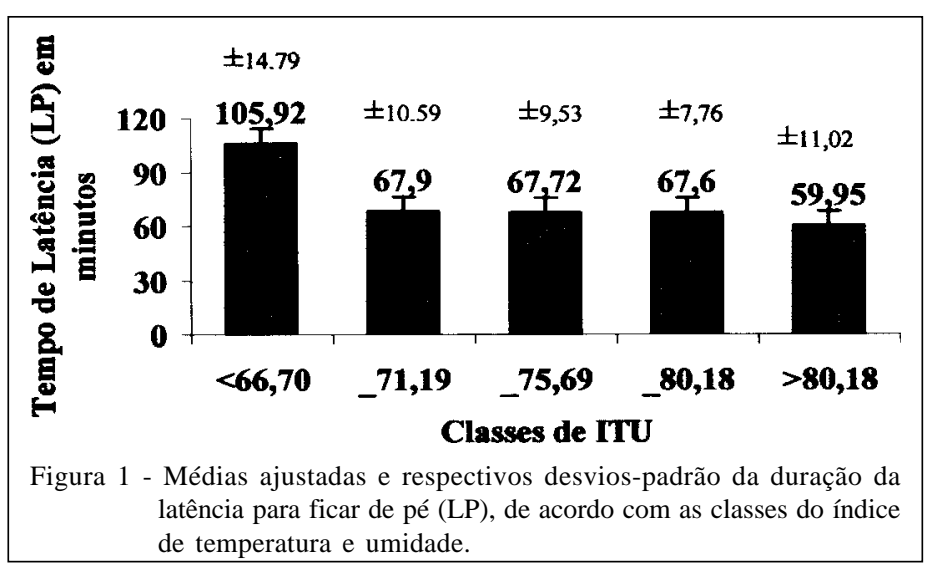

contato. Relacionando com escala evolutiva, torna-se mais fácil explicar o comportamento materno de maiores cuidados com os animais mais ágeis, já que eles, provavelmente, são potencialmente mais viáveis para se desenvolverem, e um maior investimento não seria em vão. CROMBERG et al. (1997) analisaram a freqüência com que os bezerros mudam de comportamento e as atividades desenvolvidas pelas mães, observando que animais que se movimentavam mais recebiam mais contato de suas mães.

Ciência Rural, v.37, n.5, set-out, 2007. 
Deve-se enfatizar ainda que outros aspectos dos neonatos, além da movimentação, atraem a vaca. Provavelmente, a presença de membranas e fluidos amnióticos, exalando algum odor característico, atraem a atenção da mãe, o que se comprova nos registro de observações de natimortos, casos em que as fêmeas executaram comportamentos de lamber, cheirar, empurrar e mesmo de defesa contra possíveis predadores.

\section{CONCLUSÕES}

O tempo de latência para ficar em pé representa uma boa medida de agilidade dos bezerros de corte, porém os efeitos ambientais, como espaço físico e temperatura e umidade do ar, podem afetar diretamente esses comportamentos. Considerando que o atraso na LP contribuiu para aumentar o período de latência para o bezerro ingerir o colostro, tem-se a dimensão da importância biológica que as variações de ITU podem acarretar. Na avaliação precoce de bezerros, deve-se tomar cuidado ao comparar desempenhos, pois esses podem ser prejudicados em virtude das condições ambientais desfavoráveis no momento do parto. A seleção de animais mais ágeis ao nascer e de vacas com maiores cuidados maternais, além de maior habilidade materna, definida esta como maior produção de leite para desmamar bezerros mais pesados, podem otimizar e tornar economicamente mais viável a exploração de bovinos de corte.

\section{REFERÊNCIAS}

BACCARI, F.J. Manejo ambiental da vaca leiteira em ambientes quentes. Londrina: UEL, 2001. 142p.

BLUM, J.W.; HAMMON, H. Colostrum effects on the gastrointestinal tract, and on nutritional, endocrine and metabolic parameters in neonatal calves. Livestock Production Science, v.66, p.151-159, 2000.

CROMBERG, V.U. et al. Freqüência com que os bezerros recém nascidos mudam de comportamento e suas relações com o cuidado materno e a eficiência para a primeira mamada. In. REUNIÃO ANUAL DA SOCIEDADE BRASILEIRA DE ETOLOGIA, 15., 1997, São Carlos. Anais... São Carlos: Sociedade Brasileira de Etologia, 1997. p.395.
KELLY, C.F.; BOND, T.E. Bioclimatic factors and their measurement. In: NATIONAL ACADEMY OF SCIENCES. A guide environmental research oon animals. Washington, 1971. 123p.

LE DIVIDICH, J. et al. Cold stress. In: PHILLIPS, C.; PIGGINS, D. (Edit). Farm animals and the environment. London, 1992. 375p.

LIDFORS, L. Mother young behaviour in cattle. 1994. 71f. Thesis (Doctoral in Animal Environment and Health) University of Agricultural Sciences, Swedish.

LUCCI, C.S. Bovinos leiteiros jovens. São Paulo: Nobel/ EDUSP, 1989. 146p.

MARTIN, P.; BATESON, P. Measuring behaviour: an introductory guide. Cambridge-UK: Cambridge University, 1986. 200p.

PARANHOS DA COSTA, M.J.R.; CROMBERG, V.U. Relações materno-filiais em bovinos de corte nas primeiras horas após o parto. In: _. Comportamento materno em mamíferos: bases teóricas e aplicações aos ruminantes domésticos. São Paulo: Sociedade Brasileira de Etologia, 1998. p.215-235.

PIRES, M.F.A. et al. Alguns fatores que afetam o nível das imunoglobulinas no soro de bezerros de rebanhos leiteiros. Revista da Sociedade Brasileira de Zootecnia, v.22, n.6, p.985-992, 1993.

RILEY, D.G. et al. Genetic and nongenetic influences on vigor at birth and preweaning mortality of purebred and high percentage Brahman calves. Journal of Animal Science, v.82, p.1581-1588, 2004.

SCHMIDEK, A. Análises dos fatores genéticos e ambientais relacionados a características de vigor e qualidade materna para as raças Nelore e Guzerá. 2003. 84f. Dissertação (Mestrado em Zootecnia) - Faculdade de ciências Agrárias e Veterinárias, UNESP, Jaboticabal.

SPSS Software Products, SPSS, Chicago, IL, SPSS ${ }^{\circledR}$, Copyright (C) 2003 by SPSS Inc. Acessado em 2004. Online. Disponível na Internet: ttp://www.spss.com.

THOM, E.C. The discomfort index. Weatherwise, v.12, p.57-59, 1959.

TITTO, E.A.L. Clima: influência na produção de leite. In: SILVA, I.J.O. Ambiência na produção de leite em climas quentes. Piracicaba: FEALQ, 1998. p. 0-23. 